Check for updates

Cite this: RSC Adv., 2019, 9, 7680

Received 13th December 2018 Accepted 27th February 2019

DOI: $10.1039 / c 8 r a 10241 d$

rsc.li/rsc-advances

\section{Superconductivity of boron-doped graphane under high pressure $\dagger$}

\author{
Ya Cheng, (D) ab Xianlong Wang, ${ }^{\star a b}$ Jie Zhang, ${ }^{a}$ Kaishuai Yang, (D) ab Caoping Niu ${ }^{\text {ab }}$ \\ and Zhi Zeng*ab
}

Based on first-principles calculations, the properties of B-doped graphane under high pressure up to $380 \mathrm{GPa}$ are investigated. We find that B-doped graphane undergoes a phase transition from phase- $\alpha$ to phase- $\beta$ at $6 \mathrm{GPa}$. Different from pristine graphane (X. Wen, L. Hand, V. Labet, T. Yang, R. Hoffmann, N. W. Ashcroft, A. R. Oganov and A. O. Lyakhov, Graphane sheets and crystals under pressure, Proc. Natl. Acad. Sci. U. S. A., 2011, 108, 6833-6837), phase- $\gamma$ of B-doped graphane is kinetically unstable. The calculated superconducting transition temperature of B-doped graphane at ambient pressure is $45 \mathrm{~K}$, and pressurization can increase the transition temperature notably, e.g., $77 \mathrm{~K}$ at $100 \mathrm{GPa}$. Both the electronic states at the Fermi level and the electron-phonon coupling are mainly contributed by $\mathrm{B}-\mathrm{C}$ characteristics, indicating that the $\mathrm{B}$-doping plays a key role in the superconductivity.

\section{Introduction}

Finding high critical temperature superconductors $\left(T_{\mathrm{c}}\right)$ is one of central topics of condensed matter physics and materials science. In the last several years, important progress was achieved in hydrogen-rich compounds. In 2015, under high pressure, $\mathrm{H}_{2} \mathrm{~S}$ and $\mathrm{H}_{3} \mathrm{~S}$ was identified as high-temperature superconductors with a $T_{\mathrm{c}}$ of $80 \mathrm{~K}$ and $203 \mathrm{~K}^{1-4}$ Recently, $T_{\mathrm{c}}$ as high as $215-260 \mathrm{~K}$ was reported in the $\mathrm{La}-\mathrm{H}_{x}$ system. ${ }^{5,6}$ These hydrogen-rich compounds are conventional superconductors similar to $\mathrm{MgB}_{2}{ }^{7}$ in which the superconductivity can be explained by Bardeen-Cooper-Schrieffer (BCS) theory. ${ }^{8}$ In the conventional superconductors, higher vibrational frequency generally gives higher $T_{\mathrm{c}}$. Since hydrogen is the lightest element, hydrogen in the solid metallic phase will have a high Debye frequency leading to above $3000 \mathrm{~K}$ Debye temperature., ${ }^{\mathbf{9} 10}$ In addition to hydrogen, carbon in the solid phase can have a Debye temperature of as high as $2230 \mathrm{~K},{ }^{11}$ and it usually forms networks of covalent bonds, such as graphene and diamond. Therefore, high $T_{\mathrm{c}}$ can also be expected in metallized carbon.

Since boron (B) has one electron less than carbon, B dopant is an effective way to realize the metallization of carbon networks with hole-doping. ${ }^{12-16}$ Boron dopant is also widely used to extend the applications of carbon materials, for example, B doped graphene has important applications in the fields of lithium storage ${ }^{17,18}$ and catalysts ${ }^{19}$ Furthermore, since

${ }^{a}$ Key Laboratory of Materials Physics, Institute of Solid State Physics, Chinese Academy of Sciences, Hefei 230031, China.E-mail:xlwang@theory.issp.ac.cn; zzeng@theory. issp.ac.cn

${ }^{b}$ University of Science and Technology of China, Hefei 230026, China

$\dagger$ Electronic supplementary information (ESI) available. See DOI: 10.1039/c8ra10241d the electronic states of the B-C sigma bonds will cross the Fermi level. For example, B dopant can turn diamond into superconductor with the $T_{\mathrm{c}}$ of $\sim 4 \mathrm{~K},{ }^{12}$ where sigma bond plays an important role. ${ }^{20}$ The p-type doping in organic materials can induce superconductivity, such as diamond-like crystalline hydrocarbon, ${ }^{21}$ hydrogenated carbon nanostructures, ${ }^{22}$ and polyethylene, ${ }^{23}$ and they all have noticeable superconducting transition temperatures. Actually, in the carbon based lowdimensional materials, such as graphene and carbon nanotubes, ${ }^{15,24,25}$ much stronger electron-phonon coupling (EPC) has been proved. Theoretical results show that B-doped graphene will have high $T_{\mathrm{c}}$ ranging from $49 \mathrm{~K}$ to $72 \mathrm{~K} .{ }^{25}$ Furthermore, based on the uniform hole-doping model by removing electrons from the system, maximum value of $T_{\mathrm{c}}=96 \mathrm{~K}$ was reported in hole-doped graphane at ambient pressure. ${ }^{\mathbf{1 6}}$

In spite of doping, pressure is another important and effective route to achieve higher $T_{\mathrm{c}}$ in the conventional superconductors, for example, pressure-induced high $T_{\mathrm{c}}$ in $\mathrm{H}_{x} \mathrm{~S}$ systems., ${ }^{\mathbf{1 2 , 4 , 2 6 , 2 7}}$ This is partially because that pressure can increase the phonon frequencies and induce some new phases. However, the effects of pressure on the superconductivity behaviors of carbon based two-dimensional materials have not been clarified yet. Does the pressure can also increase the $T_{\mathrm{c}}$ of carbon based two-dimensional materials notably?

In this work, we investigate the superconductivity of B-doped graphane under high pressure. The phase transition of B-doped graphane under high pressure is examined at first. Then, the properties of superconductivity are analyzed, which shows that the $T_{\mathrm{c}}$ of B-doped graphane can be notably increased from $45 \mathrm{~K}$ to $77 \mathrm{~K}$ by pressure. The discrepancies between chemical doping (B dopant) and ideal uniform hole-doping (remove electrons from the system $)^{16}$ has been clarified, where localized hole distribution 
exists in the case of B dopant but even (homogeneous) hole distribution occurs in the ideal uniform hole-doping model.

\section{Methods}

Previous simulations pointed out that for graphane, there are four stable phases with different carbon ring configurations (chair-like or boat-like) and $\mathrm{C}-\mathrm{H}$ bond orientations. ${ }^{28}$ These graphane phases are more stable than benzene from ambient pressure to high pressure $(300 \mathrm{GPa}),{ }^{29}$ and the enthalpies of several $\mathrm{CH}$ phases are shown Fig. $\mathrm{S} 1 \dagger$ as a function of pressure. We introduce $12.5 \mathrm{~mol} \% \mathrm{~B}$ dopants in these four stable phases, which are shown in the Fig. 1 as phase- $\alpha$, phase- $\beta$, phase- $\gamma$, and phase- $\delta$, respectively. Phase- $\alpha$ and phase- $\beta$ are chair-like configurations. Seeing from the right panel of Fig. 1(a), neighboring $\mathrm{C}-\mathrm{H}$ bonds locate at opposite side in phase- $\alpha$. However, in phase- $\beta$ (Fig. 1(b)), three adjacent $\mathrm{C}-\mathrm{H}$ bonds orientate to the same direction, and the other three $\mathrm{C}-\mathrm{H}$ bonds orientate in opposite directions. The other two phases are boat like, orientations of $\mathrm{C}-\mathrm{H}$ bonds or $\mathrm{B}-\mathrm{H}$ bonds are displayed in the sketches of each phase in the right part of Fig. 1.

Structural optimization, enthalpies, and electronic structures were calculated using the Vienna ab initio simulation (VASP) $\operatorname{code}^{30-32}$ with projector-augmented plane-wave (PAW) potentials. We employed $1 \mathrm{~s}^{1}, 2 \mathrm{~s}^{2} 2 \mathrm{p}^{2}, 2 \mathrm{~s}^{2} 2 \mathrm{p}^{1}$ valence states for $\mathrm{H}$, $\mathrm{C}$, and $\mathrm{B}$ potential, respectively. The energy cutoff was set to $800 \mathrm{eV}$ in all calculations, and $2 \times 2 \times 2$ supercells of graphane were used. The $k$-point grid was generated by Monkhorst-Pack scheme and ensured that the energies converged to within 1 meV per atom. The forces were converged to less than $0.001 \mathrm{eV}$ (a)
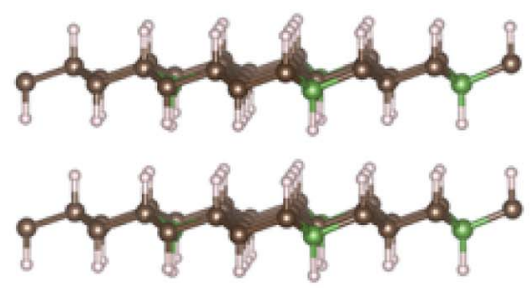

(b)

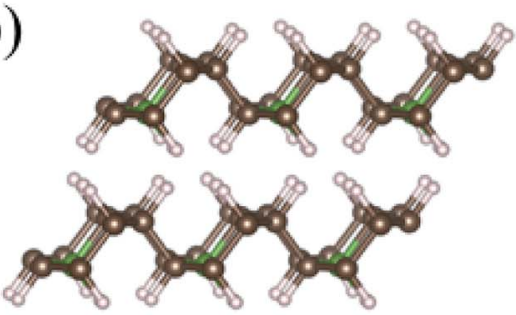

(c)

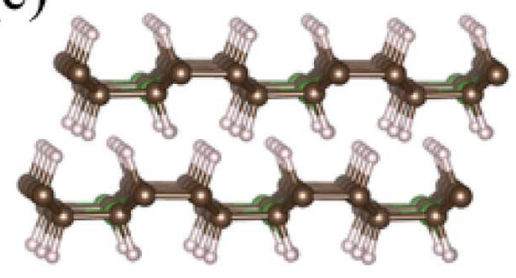

(d)

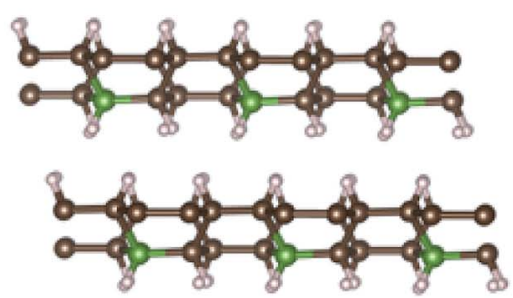

side view
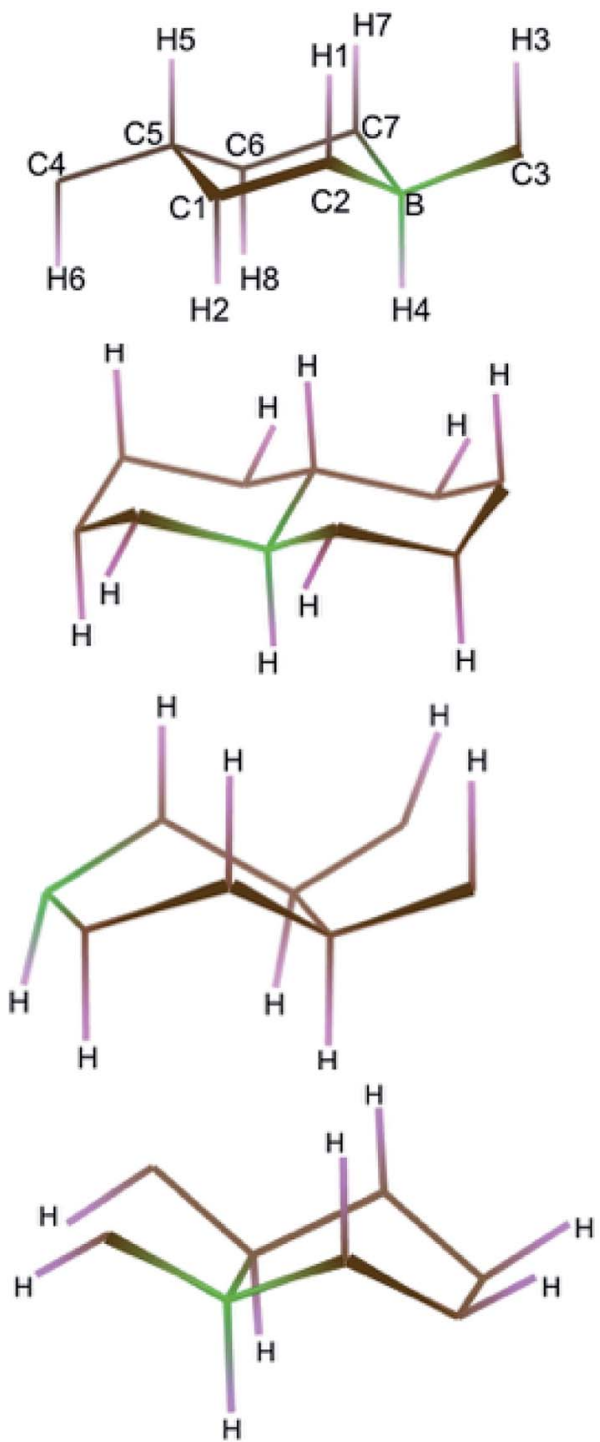

sketch

Fig. 1 Configurations of B-doped graphane in AA-stacking phase- $\alpha$ (a), $-\beta$ (b), $-\gamma(c)$, and $-\delta$ (d). Left part: side view. Right part: sketch diagram. C, $H$, and $B$ atoms are shown in brown, pink, and green, respectively. In the sketch of phase- $\alpha$, the atom positions are marked with Arabic numerals. 


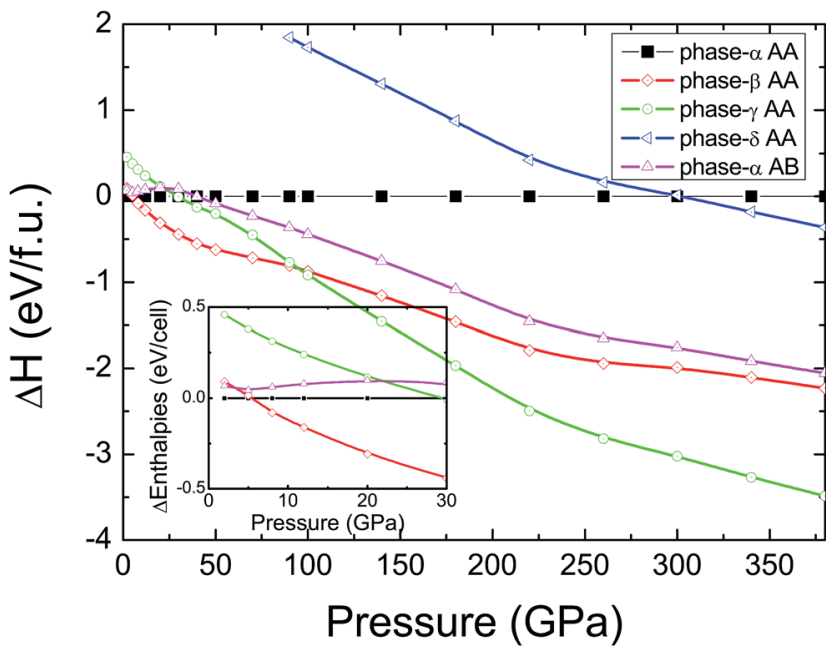

Fig. 2 Calculated enthalpies per $\mathrm{C}_{7} \mathrm{BH}_{8}$ cell as function of pressure are shown by taking the enthalpy of phase- $\alpha$ in AA-stacking as reference. $A A(A B)$ indicates the $A A(A B)$-stacking profile. The inset exhibits the zoom in image of low pressure part ( $0 \mathrm{GPa}<P<30 \mathrm{GPa})$.

$\AA^{-1}$. EPC were calculated using Quantum Espresso (QE) code $^{33}$ with density-functional perturbation theory (DFPT) ${ }^{34}$ The cutoff energies for wave functions and charge densities were set to 40 Ry and $240 \mathrm{Ry}$, respectively. We used $2 \times 2 \times 2 q$-point meshes for EPC parameter $\lambda$, and denser $k$-point meshes $8 \times 8 \times 8$ for electron-phonon interaction matrix element calculation.

\section{Results and discussion}

In pristine bulked graphane, AA-stacking features were proved to be more stable than AB-stacking cases. ${ }^{28}$ To check the stability of AA-stacking or AB-stacking in B-doped graphane, we calculated enthalpies of phase- $\alpha$ in AA-stacking and in AB-stacking. The calculated enthalpies are shown in Fig. 2. The inset of Fig. 2 zooms in low pressure range, and it shows that the enthalpy of phase- $\alpha$ in AA-stacking is smaller than that of phase- $\alpha$ in AB-stacking, indicating that similar to pristine graphane, B-doped graphane in ABstacking is metastable below $40 \mathrm{GPa}$. Therefore, we will only consider the stability of phase- $\alpha$, phase- $\beta$, phase- $\gamma$, and phase- $\delta$ in AA-stacking profiles, and the calculated enthalpies of these phases are shown in Fig. 2 as function of pressure.

We can find from Fig. 2 that the most stable phase in the pressure range 0-6 GPa is the phase- $\alpha$ in AA-stacking, which has a symmetry of $P 3 m 1$. Above $6 \mathrm{GPa}$, phase- $\beta$ becomes more stable than phase- $\alpha$, and phase- $\beta$ keeps the stability up to 95 GPa. Upon further compression, phase- $\gamma$ has the lowest enthalpy at the pressure higher than $95 \mathrm{GPa}$. Phase- $\delta$ is not competitive in enthalpy with other configurations in the whole pressure range from 0 to $380 \mathrm{GPa}$. Moreover, imaginary frequencies are observed in the phonon dispersions of phase- $\gamma$, indicating that it is kinetically unstable. Therefore, for B-doped graphane, phase- $\alpha$ (0-6 GPa) and phase- $\beta$ (6-380 GPa) are two stable phases under pressure. The phase transition of B-doped graphane is different from that of pristine, which undergoes phase transition from phase- $\alpha$ to phase- $\beta$ at 15 GPa then to phase- $\gamma$ at $240 \mathrm{GPa} .{ }^{28}$ For comparison, we calculated the phase transition of $6.125 \mathrm{~mol} \% \mathrm{~B}$ dopants in these three phases (see in Fig. S2 $\dagger$ ), we can find that the phase transition sequence of $6.125 \mathrm{~mol} \% \mathrm{~B}$-doped graphane is constant with the case of $12.5 \mathrm{~mol} \%$-doped case. The critical pressure of $6.125 \mathrm{~mol} \% \mathrm{~B}$ doped graphane from phase- $\alpha$ to phase- $\beta$ is $11 \mathrm{GPa}$, then transform to phase- $\gamma$ at $123 \mathrm{GPa}$. The critical pressure points fall in between pristine and $12.5 \mathrm{~mol} \% \mathrm{~B}$ dopants graphane.

With pressure increasing, distance between atoms will change, and the results are summarized in Table $\mathrm{S} 1, \dagger$ will change. The B-H bond length $\left(d_{\mathrm{B}-\mathrm{H}}\right)$ in B-doped graphane keeps decreasing from $1.208 \AA$ (phase- $\alpha$ at ambient pressure) to 1.150 $\AA$ (phase- $\beta$ at $100 \mathrm{GPa})$. On the other hand, $\mathrm{C}-\mathrm{H}$ bond length $\left(d_{\mathrm{C}-\mathrm{H}}\right)$ is slightly shorter $(0.1 \AA)$ than $d_{\mathrm{B}-\mathrm{H}}$ under pressure. From
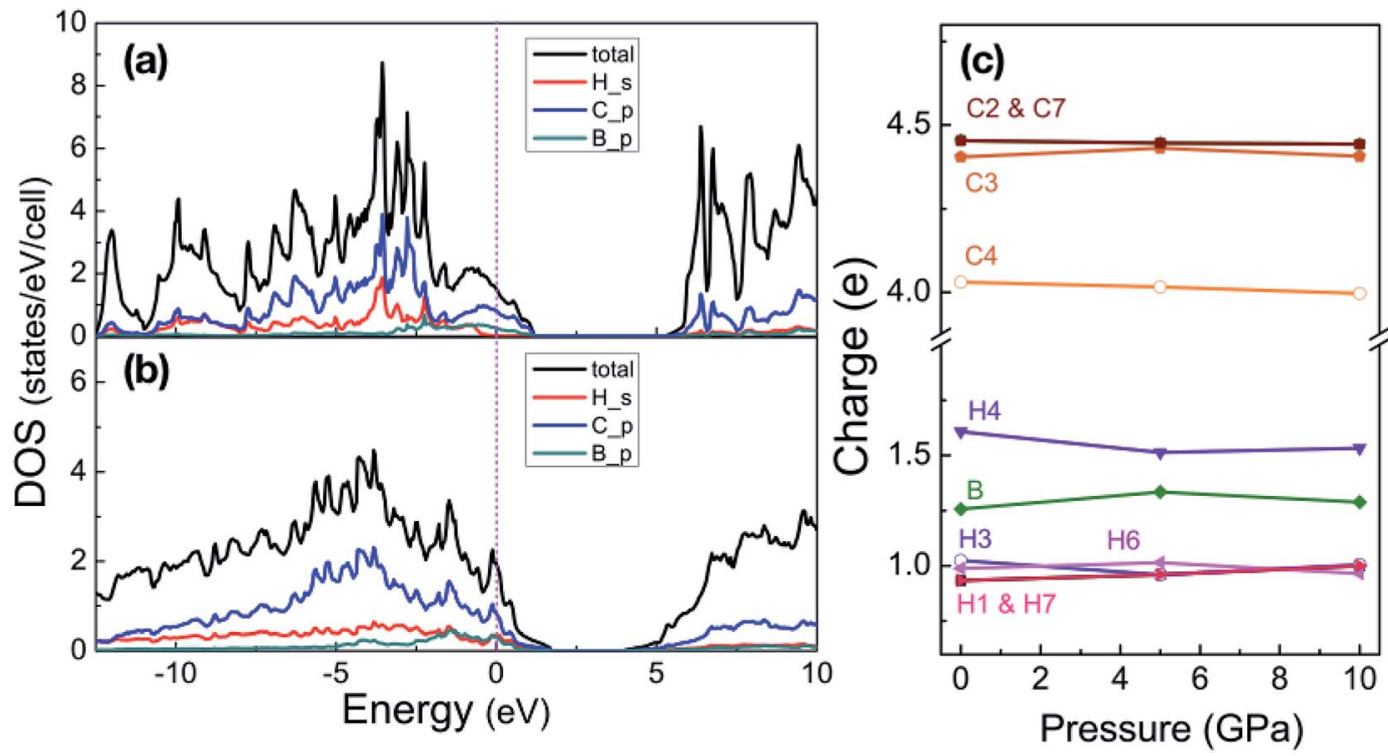

Fig. 3 Partial density of states of phase- $\alpha$ at 5 GPa (a) and phase- $\beta$ at $100 \mathrm{GPa}$ (b). Energy is respected to the Fermi level. (c) Shows the Bader charges of atoms in the phase- $\alpha$ are shown as a function of pressure, the numbers match the sketch of phase- $\alpha$ in Fig. 1 . 
Table 1 Superconducting properties of B-doped graphane under pressure. $\mathrm{N}_{\mathrm{EF}}$ is the electronic states at the Fermi level

\begin{tabular}{|c|c|c|c|c|c|c|}
\hline \multirow[b]{2}{*}{ Phases } & \multirow{2}{*}{$\begin{array}{l}\text { Pressure } \\
\text { (GPa) }\end{array}$} & \multirow[b]{2}{*}{$\lambda$} & \multirow{2}{*}{$\begin{array}{l}\mathrm{N}_{\mathrm{EF}} \\
\text { (states per cell) }\end{array}$} & \multirow{2}{*}{$\begin{array}{l}\omega_{\log } \\
(\mathrm{K})\end{array}$} & \multicolumn{2}{|l|}{$T_{\mathrm{c}}(\mathrm{K})$} \\
\hline & & & & & $\mu^{*}=0.10$ & $\mu^{*}=0.13$ \\
\hline \multirow{3}{*}{$\begin{array}{l}\text { Phase- } \\
\alpha\end{array}$} & 0 & 1.62 & 9.66 & 369 & 45 & 41 \\
\hline & 5 & 1.52 & 9.75 & 588 & 68 & 62 \\
\hline & 10 & 1.26 & 9.53 & 730 & 69 & 62 \\
\hline \multirow{3}{*}{$\begin{array}{l}\text { Phase- } \\
\beta\end{array}$} & 20 & 0.92 & 8.25 & 644 & 39 & 33 \\
\hline & 50 & 1.00 & 10.16 & 718 & 50 & 43 \\
\hline & 100 & 1.72 & 10.96 & 602 & 77 & 71 \\
\hline
\end{tabular}

Bader charge analysis of phase- $\alpha$ (see Fig. 3(c)), we can see that the electrons of $\mathrm{B}$ atom transform to neighbor atoms ( $\mathrm{H}$ and $\mathrm{C}$ ), since the electronegativity of $\mathrm{B}$ is weaker than $\mathrm{C}$ and $\mathrm{H}$. The length of $\mathrm{C}-\mathrm{C}$ bond next to $\mathrm{B}$ atom is shorter than that of $\mathrm{C}-\mathrm{C}$ bond far from $\mathrm{B}$ atom, because of the charge transformation of $\mathrm{B}$ to C. Furthermore, over the whole investigated pressure range, B-doped graphane crystal keeps layer stacking and the layer distance represented as $d_{\mathrm{H}-\mathrm{H}}$ in Table $\mathrm{S} 1 \uparrow$ decreases with pressure increasing.

We now inspect the electronic structure of the most stable phases - phase- $\alpha$ and phase- $\beta$. The partial density of states (PDOS) of phase- $\alpha$ at 5 GPa and phase- $\beta$ at 100 GPa shown in Fig. 3(a) and (b) illustrate that B-doped graphane in both phase$\alpha$ and phase- $\beta$ are metal. The electrons of $\mathrm{C}$ p-orbitals and $\mathrm{B}$ porbitals make the most contribution to the density of states but not those of $\mathrm{H}$ s-orbitals indicating that the metallization origins from the $\mathrm{C}-\mathrm{B}$ rings. Moreover, the $\mathrm{H}$ s-orbitals electrons contribute to the superconductivity of phase- $\beta$, since $\mathrm{H} \mathrm{s}$ orbitals cross the Fermi level. As shown in Table 1, density of states at the Fermi level increases slightly with pressure increasing in phase- $\beta$, e.g., the electronic states increase from 8.50 states per cell at $20 \mathrm{GPa}$ to 10.96 states per cell at $100 \mathrm{GPa}$.
The phonon dispersion curves of phase- $\alpha$ (see Fig. S2 $\dagger$ ) and phase- $\beta$ (see Fig. 4) under pressure were calculated to explore the lattice dynamics of B-doped graphane. The absence of imaginary frequencies implies the kinetically stability of phase$\beta$ under high pressure. The whole phonon density of states of phase- $\alpha$ at $10 \mathrm{GPa}$ can be divided into three parts, which is shown in Fig. 5(a). The low-frequency vibration below $750 \mathrm{~cm}^{-1}$ mainly comes from the $\mathrm{C}-\mathrm{C}$ or $\mathrm{C}-\mathrm{B}$ stretching vibrations, $\mathrm{C}-\mathrm{H}$ and $\mathrm{B}-\mathrm{H}$ bonds shear motions contribute to the middlefrequency vibration (750-1500 $\left.\mathrm{cm}^{-1}\right) .{ }^{16}$ After a large gap, $\mathrm{C}-\mathrm{H}$ and $\mathrm{B}-\mathrm{H}$ bonds stretching mainly contribute to the highest frequency area above $2500 \mathrm{~cm}^{-1}$. Among this area, B-H stretching vibration appears around $2600 \mathrm{~cm}^{-1}$, while $\mathrm{C}-\mathrm{H}$ stretching mode sites around $3000 \mathrm{~cm}^{-1}$. Phase- $\alpha$ and phase$\beta$ at all calculated pressure points have similar profiles in phonon dispersion, all of them can be divided into three parts in frequency.

Taking phase- $\beta$ at $100 \mathrm{GPa}$ for example, from the Eliashberg phonon spectral function $\alpha^{2} F(\omega)$ and integrated EPC parameter $(\lambda)$ as shown in Fig. 5(b), the low-frequency (blow $750 \mathrm{~cm}^{-1}$ ) and (750-1500 $\mathrm{cm}^{-1}$ ) middle-frequency vibration contributes as much as $50.1 \%$ and $38.0 \%$ of total electron-phonon coupling constant, respectively. The remaining $\lambda$ comes from highfrequency $\mathrm{C}-\mathrm{H}$ and $\mathrm{B}-\mathrm{H}$ stretching motions. This result indicates the significant role of low-frequency and middlefrequency vibrational modes. However, C-H and B-H stretching motions contribute less to electron-phonon interaction. Namely, B-C rings play an important role in the superconductivity of B-doped graphane.

Based on the $\lambda$ and logarithm of average frequency $\left(\omega_{\log }\right)$, we employed the modified McMillan equation by Allen and Dynes to calculate the $T_{\mathrm{c}}$.

$$
T_{\mathrm{c}}=\frac{\omega_{\log }}{1.2} \exp \left(-\frac{1.04(1+\lambda)}{\lambda-\mu^{*}(1+0.62 \lambda)}\right),
$$

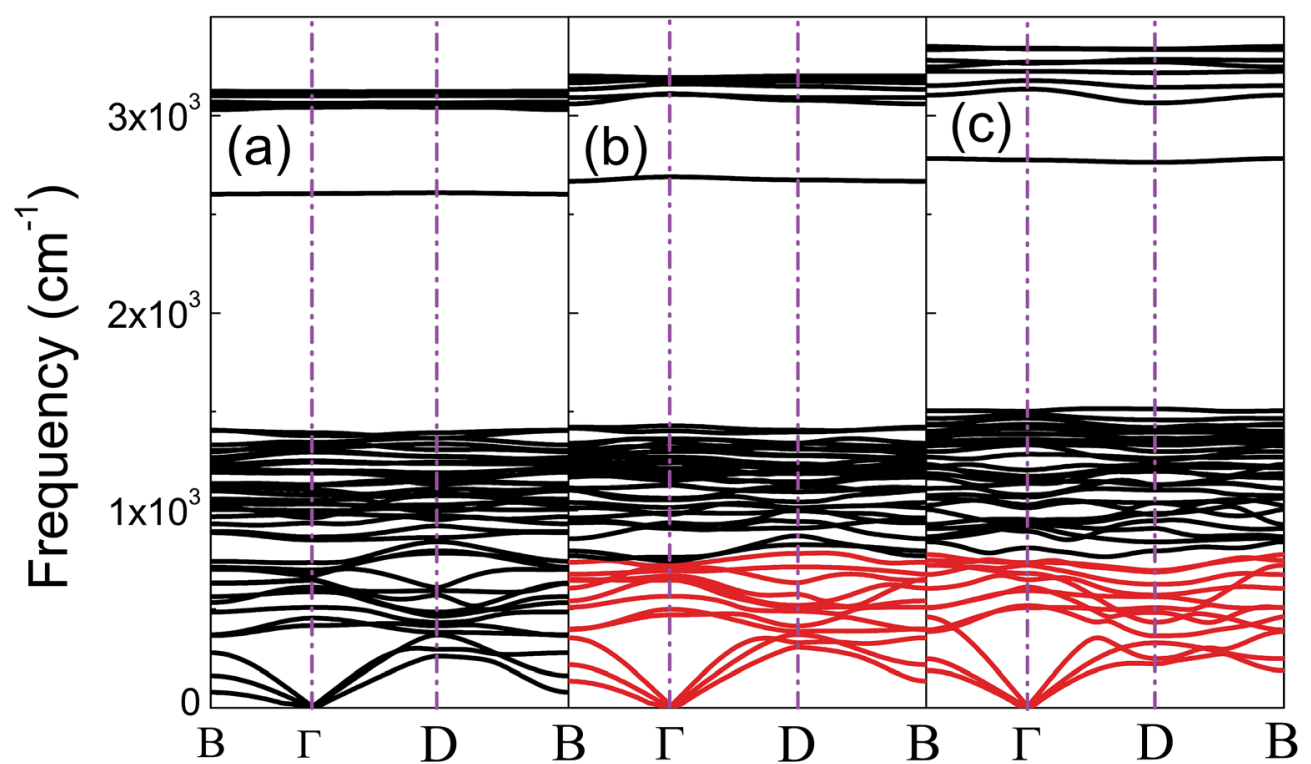

Fig. 4 (a), (b), and (c) show the phonon dispersions of phase- $\beta$ at $20 \mathrm{GPa}, 50 \mathrm{GPa}$, and $100 \mathrm{GPa}$, respectively. The softened phonon branches at D point are shown in red at (b) and (c). 

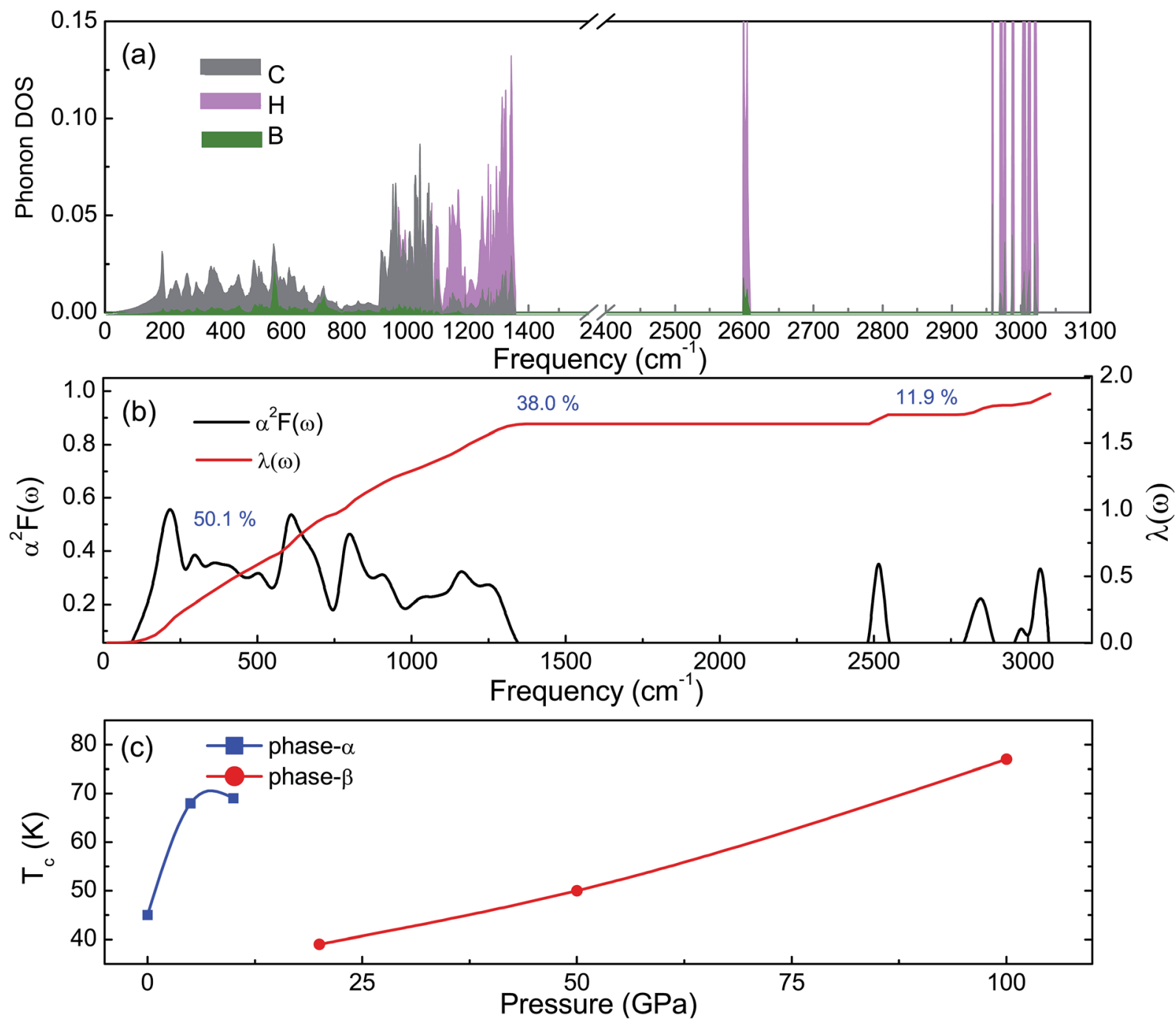

Fig. 5 (a) Partial phonon density of states of graphane at $10 \mathrm{GPa}$. Gray, pink, and green represent $\mathrm{C}, \mathrm{H}$, and B atoms, respectively. (b) Shows the Eliashberg phonon spectral function $\alpha^{2} F(\omega)$ and electron-phonon integral $\lambda(\omega)$ of phase- $\beta$ at $100 \mathrm{GPa}$. (c) Shows the $T_{\mathrm{c}}$ of B-doped graphane is shown as function of pressure.

where $\mu^{*}$ stands for the screened Coulomb repulsion between paired electrons, ${ }^{9}$ and it is typically chosen between $0.10-0.15$. We chose $\mu^{*}$ value 0.10 and 0.13 in this work. The calculated parameters related to superconductivity are listed in Table 1 , where $\mathrm{N}_{\mathrm{EF}}$ represents the electronic states at the Fermi level. We can find that the $\mathrm{N}_{\mathrm{EF}}$ of phase- $\alpha$ at $10 \mathrm{GPa}$ is smaller than that of $5 \mathrm{GPa}$, but larger than that of phase- $\beta$ at $20 \mathrm{GPa}$. Since the electrons of $\mathrm{H}$ s-orbital contribute to $\mathrm{N}_{\mathrm{EF}}$, the $\mathrm{N}_{\mathrm{EF}}$ of phase- $\beta$ at $50 \mathrm{GPa}$ and $100 \mathrm{GPa}$ are much larger than that of phase- $\alpha$ under pressure. Take phase- $\beta$ at $100 \mathrm{GPa}$ for instance, the $\lambda$ is 1.72 and $\omega_{\log }$ is $602 \mathrm{~K}$, leading to that the calculated $T_{\mathrm{c}}$ using modified McMillan equation is $77 \mathrm{~K}$.

To clarify the pressure effects on the superconductivity of Bdoped graphane, we calculated the critical temperature of superconductivity of both phases. The $T_{\mathrm{c}}$ of B-doped graphane at the pressure ranging from ambient pressure to $100 \mathrm{GPa}$ was calculated, and the results are presented in Fig. 5(c). At ambient pressure, the $T_{\mathrm{c}}$ of phase- $\alpha$ is $45 \mathrm{~K}$, while it increases to $68 \mathrm{~K}$ at the pressure of $5 \mathrm{GPa}$. The $T_{\mathrm{c}}$ at $10 \mathrm{GPa}(69 \mathrm{~K})$ is comparable with that of $5 \mathrm{GPa}$, and the reason is that from $5 \mathrm{GPa}$ to $10 \mathrm{GPa}$, the decrease of $\lambda$ counteracts the increase of $\omega_{\log }$ as shown in Table 1. It is noticeable that the $T_{\mathrm{c}}$ of phase- $\alpha$ we simulated at ambient pressure is $45 \mathrm{~K}$, much lower than that (96 K) predicted by Savini $e t$ al. ${ }^{16}$ The reason lead to this large divergence mainly comes from the charge distribution difference between chemical doping and ideal uniform electronic doping. In the ideal uniformed electronic doping model by removing electrons from the investigated system, due to the nesting of the Fermi surface, large medium frequency phonon soften appears at gamma point. ${ }^{16}$ As shown in Fig. S4, $\dagger$ we obtain similar phonon soften in the graphane by removing $12.5 \%$ electrons. However, in the case of chemical doping, the charge is localized around B atom but not uniformly distributed in real chemical doping mode of phase- $\alpha$. It can be found in Fig. 3(c) that charges of B atom transform to atoms nearby $(\mathrm{C}$ and $\mathrm{H})$. That is the reason that we did not find the medium frequency phonon soften at gamma 
point as shown in Fig. 4. In phase- $\beta$, the $\omega_{\log }$ of phase- $\alpha$ at $20 \mathrm{GPa}$ and $50 \mathrm{GPa}$ is $644 \mathrm{~K}$ and $718 \mathrm{~K}$, respectively. However, it decreases to $602 \mathrm{~K}$ at the pressure of $100 \mathrm{GPa}$. The decrease of $\omega_{\log }$ from $50 \mathrm{GPa}$ to $100 \mathrm{GPa}$ origins from the soften of phonon at the D point (Fig. 4 (b) and (c)) induced by pressure. The pressure-induced phonon softening is a common behavior of superconductors at high pressure, ${ }^{35-37}$ and it plays a crucial role in enhancing the electron-phonon coupling. ${ }^{1,38,39}$ Therefore, the $\lambda$ of phase- $\beta$ increases with the pressure increasing, which counteracts the decrease of $\omega_{\log }$. We can find that, over the pressure range from $20 \mathrm{GPa}$ to $100 \mathrm{GPa}$, the $T_{\mathrm{c}}$ of phase- $\beta$ almost monotonously increases from $39 \mathrm{~K}$ to $77 \mathrm{~K}$. Our results show that the $T_{\mathrm{c}}$ of each $\mathrm{B}$-doped graphane phase will notably increase with pressure increasing. The B-C bonds make the most contribution to $T_{\mathrm{c}}$ in both phase- $\alpha$ and phase- $\beta$, and upon further compression, $\mathrm{H}$ s-orbitals electrons in the phase- $\beta$ will contribute more to the metallization and superconductivity of B-doped graphane. For the lower doping concentration, e.g., $6.125 \mathrm{~mol} \%$ B-doped graphane, the electronic states at the Fermi level will become smaller. Therefore, similar to the concentration effect of hole-doped graphane, ${ }^{\mathbf{1 6}}$ lower B concentration may lead to lower superconducting critical temperature.

\section{Conclusions}

We have investigated the phase transition, metallization, and superconductivity of B-doped graphane in the framework of density functional theory. We found that at low pressure (0-6 $\mathrm{GPa}$ ), B-doped graphane in phase- $\alpha$ with AA-stacking is the most stable phase, and AB-stacking are metastable configuration. Phase- $\beta$ is the most stable case within the pressure ranging from 6 to $380 \mathrm{GPa}$. All B-doped graphane phases are metal, and $\mathrm{B}-\mathrm{C}$ and $\mathrm{C}-\mathrm{C}$ vibrations dominantly contribute to the electronphonon coupling. At ambient pressure, because of the Fermi surface nesting induced phonon softening, the ideal uniform hole-doping model by removing electrons from graphane gives higher $T_{\mathrm{c}}$ than that of the B-doped case. Moreover, pressureinduced phonon softening in B-doped graphane is observed, and pressure can significantly increase the $T_{\mathrm{c}}$ of B-doped graphane in both phase- $\alpha$ and phase- $\beta$.

\section{Conflicts of interest}

There are no conflicts to declare.

\section{Acknowledgements}

This research was supported by the NSFC under Grant of 11674329, Science Challenge Project No. TZ2016001, the National Science Foundation for Young Scientists of China (Grant No. 11504381), and the Major/Innovative Program of Development Foundation of Hefei Center for Physical Science and Technology. The calculations were partly performed in Center for Computational Science of CASHIPS, the ScGrid of Supercomputing Center and Computer Network Information Center of Chinese Academy of Sciences.

\section{References}

1 Y. Li, J. Hao, H. Liu, Y. Li and Y. Ma, The metallization and superconductivity of dense hydrogen sulfide, J. Chem. Phys, 2014, 140, 174712.

2 D. Duan, X. Huang, F. Tian, D. Li, H. Yu, Y. Liu, Y. Ma, B. Liu and T. Cui, Pressure-induced decomposition of solid hydrogen sulfide, Phys. Rev. B: Condens. Matter Mater. Phys., 2015, 91, 180502.

3 D. Duan, Y. Liu, F. Tian, D. Li, X. Huang, Z. Zhao, H. Yu, B. Liu, W. Tian and T. Cui, Pressure-induced metallization of dense $\left(\mathrm{H}_{2} \mathrm{~S}\right)_{2} \mathrm{H}_{2}$ with high- $\mathrm{T}_{\mathrm{c}}$ superconductivity, Sci. Rep., 2014, 4, 6968.

4 A. P. Drozdov, M. I. Eremets, I. A. Troyan, V. Ksenofontov and S. I. Shylin, Conventional superconductivity at 203 kelvin at high pressures in the sulfur hydride system, Nature, 2015, 525, 73-76.

5 A. P. Drozdov, V. S. Minkov, S. P. Besedin, P. P. Kong, M. A. Kuzovnikov, D. A. Knyazev and M. I. Eremets, Superconductivity at $215 \mathrm{~K}$ in lanthanum hydride at high pressures, 2018, arXiv: 1808.07039.

6 M. Somayazulu, M. Ahart, A. K. Mishra, Z. M. Geballe, M. Baldini, Y. Meng, V. V Struzhkin and R. J. Hemley, Evidence for superconductivity above $260 \mathrm{~K}$ in lanthanum superhydride at megabar pressures, 2018, arXiv: 1808.07695.

7 J. Nagamatsu, N. Nakagawa, T. Maranaka, Y. Zenitani and J. Akimitsu, Superconductivity at $39 \mathrm{~K}$ in copper doping magnesium diboride, Nature, 2001, 410, 63-64.

$8 \mathrm{~J}$. Bardeen, L. N. Cooper and J. R. Schrieffer, Theory of superconductivity, Phys. Rev., 1957, 108, 1175-1204.

9 N. W. Ashcroft, Metallic hydrogen: a high-temperature superconductor?, Phys. Rev. Lett., 1968, 21, 1748-1749.

10 V. L. Ginzburg, The Physics of a Lifetime: Reflections on the Problems and Personalities of 20th Century Physics, Springer, 2001.

11 C. Kittel, Introduction to Solid State Physics, John Wiley \& Sons, 8 edn, 2004.

12 E. A. Ekimov, V. a Sidorov, E. D. Bauer, N. N. Mel'nik, N. J. Curro, J. D. Thompson and S. M. Stishov, Superconductivity in diamond, Nature, 2004, 428, 542-545.

13 E. A. Ekimov, V. A. Sidorov, A. Zoteev, Y. Lebed, J. D. Thompson, E. D. Bauer and S. M. Stishov, Superconductivity in diamond induced by boron doping at high pressure, Phys. Status Solidi B, 2009, 246, 667-672.

14 H. Fukuyama, Y. Ohta, T. Shirakawa and S. Horiuchi, Superconductivity in B-doped diamonds, J. Phys. Chem. Solids, 2008, 69, 3265-3268.

15 A. Y. Kasumov, M. Kociak, M. Ferrier, Y. A. Kasumov, S. Gueron, B. Reulet, I. I. Khodos, Y. B. Gorbatov, V. T. Volkov, L. Vaccarini and H. Bouchiat, Superconductivity in ropes of single-walled carbon nanotubes, Phys. Rev. Lett., 2001, 86, 2416-2419.

16 G. Savini, A. C. Ferrari and F. Giustino, First-principles prediction of doped graphane as a high-temperature electron-phonon superconductor, Phys. Rev. Lett., 2010, 105, 037002. 
17 D. Das, R. P. Hardikar, S. S. Han, K. R. Lee and A. K. Singh, Monolayer BC2: an ultrahigh capacity anode material for $\mathrm{Li}$ ion batteries, Phys. Chem. Chem. Phys., 2017, 19, 2423024239.

18 X. Wang, Z. Zeng, H. Ahn and G. Wang, First-principles study on the enhancement of lithium storage capacity in boron doped graphene, Appl. Phys. Lett., 2009, 95, 183103.

19 J. Y. Damte, S. L. Lyu, E. G. Leggesse and J. C. Jiang, Methanol decomposition reactions over a boron-doped graphene supported Ru-Pt catalyst, Phys. Chem. Chem. Phys., 2018, 20, 9355-9363.

$20 \mathrm{~J}$. M. An and W. E. Pickett, Superconductivity of $\mathrm{MgB}_{2}$ : covalent bonds driven metallic, Phys. Rev. Lett., 2001, 86, 4366-4369.

21 C. Lian, J. Wang, W. Duan and C. Chen, superconductivity in hole- doped diamond-like crystalline hydrocarbon, Sci. Rep., 2017, 7, 1-6.

22 A. Sanna, A. Davydov, J. K. Dewhurst and S. Sharma, Superconductivity in hydrogenated carbon nanostructures, Eur. Phys. J. B, 2018, 91, 177.

23 A. F. José, M. Graužinytè, Li. Boeri, G. Profeta and A. Sanna, Superconductivity in doped polyethylene at high pressure, Eur. Phys. J. B, 2018, 91, 176.

24 F. Mazzola, J. W. Wells, R. Yakimova, S. Ulstrup, J. A. Miwa, R. Balog, M. Bianchi, M. Leandersson, J. Adell, P. Hofmann and T. Balasubramanian, Kinks in the $\sigma$ band of graphene induced by electron-phonon coupling, Phys. Rev. Lett., 2013, 111, 216806.

25 F. Mazzola, T. Frederiksen, T. Balasubramanian, P. Hofmann, B. Hellsing and J. W. Wells, Strong electronphonon coupling in the $\sigma$ band of graphene, Phys. Rev. B, 2017, 95, 075430.

26 A. F. Goncharov, S. S. Lobanov, I. Kruglov, X. Zhao, X. Chen, A. R. Oganov, Z. Konôpková and V. B. Prakapenka, Hydrogen sulfide at high pressure: change in stoichiometry, Phys. Rev. $B, 2016,93,174105$.

27 T. Ishikawa, A. Nakanishi, K. Shimizu, H. KatayamaYoshida, T. Oda and N. Suzuki, Superconducting $\mathrm{H}_{5} \mathrm{~S}_{2}$ phase in sulfur-hydrogen system under high-pressure, Sci. Rep., 2016, 6, 23160.

28 X. Wen, L. Hand, V. Labet, T. Yang, R. Hoffmann, N. W. Ashcroft, A. R. Oganov and A. O. Lyakhov, Graphane sheets and crystals under pressure, Proc. Natl. Acad. Sci. U. S. A., 2011, 108, 6833-6837.
29 X. Wen and N. W. Ashcroft, Benzene under high pressure: a story of molecular crystals transforming to saturated networks, with a possible intermediate metallic phase, $J$. Am. Chem. Soc., 2011, 133, 9023-9035.

30 G. Kresse and J. Furthmüller, Efficiency of $a b$ initio total energy calculations for metals and semiconductors using a plane-wave basis set, Comput. Mater. Sci., 1996, 6, 15-50.

31 G. Kresse and J. Furthmüller, Efficient iterative schemes for $a b$ initio total-energy calculations using a plane-wave basis set, Phys. Rev. B: Condens. Matter Mater. Phys., 1996, 54, 11169-11186.

32 G. Kresse and D. Joubert, From ultrasoft pseudopotentials to the projector augmented-wave method, Phys. Rev. B: Condens. Matter Mater. Phys., 1999, 59, 1758-1775.

33 P. Giannozzi, S. Baroni, N. Bonini, M. Calandra, R. Car, C. Cavazzoni, D. Ceresoli, G. L. Chiarotti, M. Cococcioni, I. Dabo, A. Dal Corso, S. De Gironcoli, S. Fabris, G. Fratesi, R. Gebauer, U. Gerstmann, C. Gougoussis, A. Kokalj, M. Lazzeri, L. Martin-Samos, N. Marzari, F. Mauri, R. Mazzarello, S. Paolini, A. Pasquarello, L. Paulatto, C. Sbraccia, S. Scandolo, G. Sclauzero, A. P. Seitsonen, A. Smogunov, P. Umari and R. M. Wentzcovitch, QUANTUM ESPRESSO: a modular and open-source software project for quantum simulations of materials, $J$. Phys.: Condens. Matter, 2009, 21, 395502.

34 S. Baroni, S. de Gironcoli, A. D. Corso and P. Giannozzi, Phonons and related crystal properties from densityfunctional perturbation theory, Rev. Mod. Phys., 2001, 73, 515-562.

35 Y. Cheng, C. Zhang, T. Wang, G. Zhong, C. Yang, X. Chen and H. Q. Lin, Pressure-induced superconductivity in $\mathrm{H}_{2}$ containing hydride $\mathrm{PbH}_{4}\left(\mathrm{H}_{2}\right)_{2}$, Sci. Rep., 2015, 5, 16475.

36 G. Zhong, C. Yang, X. Chen and H. Lin, Superconductivity in solid benzene molecular crystal, J. Phys.: Condens. Matter, 2018, 30, 245703.

37 M. L. Cohen, Electronphonon induced pairing and its limits for superconducting systems, Phys. E, 2011, 43, 657-660.

38 P. B. Allen and R. C. Dynes, Transition temperature of strong-couple superconductors reanalyzed, Phys. Rev. B: Condens. Matter Mater. Phys., 1975, 12, 905-922.

39 N. Bernstein, C. S. Hellberg, M. D. Johannes, I. I. Mazin and M. J. Mehl, What superconducts in sulfur hydrides under pressure and why, Phys. Rev. B: Condens. Matter Mater. Phys., 2015, 91, 060511. 